Conference Article

\title{
The Physics of Interdependence, Social Uncertainty Relations, and Incompleteness
}

\author{
W.F. Lawless
}

Paine College, $123515^{\text {th }}$ Street, Augusta, GA 30901

\begin{abstract}
We report on the development of a mathematical model of social uncertainty relations to replace traditional models of the interaction, as well as a model of complexity from econophysics. Our goal with this mathematics is to control hybrid teams, firms and systems (i.e., where "hybrids" are arbitrary combinations of humans, robots and machines). But uncertainty is created by states of interdependence between social objects: at one extreme, interdependence reduces to independence between agents, producing rational but asocial effects; at the other extreme, interdependence de-individuates a group's members until individual identity dissolves into a group (e.g., strong cults, mobs, gangs, and well-run teams and firms). In other studies, we have reviewed the structure of teams; in this report, we focus on how interdependence impedes efforts at direct control by making meaning incomplete. We begin with bistability to simplify interdependence, and generalize to full interdependence.
\end{abstract}

Keywords: interdependence; uncertainty; incompleteness; computational models

\section{Introduction}

A new econophysics (e.g., [1]) model of the interaction concludes that cooperation and knowledge are key to team and organizational success, characterized by adaptability. A model by Conant and Ashby [2] concludes mathematically that the optimal organization is not adaptive to environmental pressures (viz., as an organization is with the six-sigma processes that attempt to minimize waste; in [3]), but minimizes errors, and, in the limit, has a controller that acts like the system it regulates (for others, see [4],[5]). But these models are passive to interdependence. Social systems operate in states of interdependence [6] that cause uncertainty [7], making the mathematics intractable [8], except for the formation of stable swarms or patterns (e.g., car platoons to drive on the highway). Modeling and controlling social interdependence mathematically remains unsolved (e.g., [9]). But with our mathematical model of interdependence, we propose that indirect control is possible. Our model is unfinished; we conclude with plans on how we expect it to reach closure.

\section{Interdependence in the field.}

Putting uncertainty aside until later, the effects of a community matrix $A$ can be measured in the field. Assume that competition for resources occurs within and between groups; that, unlike the inability of individuals to multitask [10],

\footnotetext{
*E-mail address: wlawless@paine.edu

ISSN: 1791-2377 @ 2015 Kavala Institute of Technology. All rights reserved.
}

multitasking is the purpose of groups [11]; and that the optimal group multitasks seamlessly, generating a baseline entropy for stable organizations that we initially, but incorrectly, set to zero [12], noting that, similarly, stable knowledge implies zero entropy [13]. We justify this assumption at this time by observing that, compared to functional groups, an individual is less able to survive (defending itself, feeding itself, etc.). That is, a collective of individuals is in a higher state of average uncertainty or agitation than the same individuals independently performing the identical actions but as part of a group (e.g., with coordination).

Competition between groups increases cooperation among ingroup members ([14],[15]), helping a group to multitask in its struggle to survive. Given $A$ as a operator that serves as a community matrix of, for example, possible cooperators working together to multi-task within a tribe's ingroup, or competitors in an outgroup, let $a_{i j}$ represent the effects of agent- $i$ on agent $-j$, the opposite for $a_{j i}$ [16] (for ingroup-outgroup effects, see [17]; for a system of tribal effects, see [18]). The strength of cooperation to multi-task can be measured by the state of interdependence in community matrix $A$, where interdependence is the effect that a group has on the choices and behaviors of its members; we designate interdependence as $\rho$ :

$$
\rho=\left(M S_{G / T}-M S_{S / G / T}\right) /\left(M S_{G / T}+(n-1) M S_{S / G / T}\right)
$$

$M S_{G / T}$ is the sum of the mean squares from the group on a measurement of an issue or problem that is a group's focus as it assigns roles that produce multitasking, represent- 
ed by $T$ (e.g., requiring multiple tasks, a plan of action to address the competition); $M S_{S / G / T}$ is the aggregated contribution from the individuals on a measurement of factor $T$; and $n$ represents the number of members in a group being measured (from [19]; see p. 235). $\rho$ ranges from -1 as multitasking goes to zero when the group is replaced by a collection of independent individuals (e.g., job seekers); or $\rho$ can range to +1 as multitasking replaces the individual with slavish subservience to a group's efforts, to a charismatic leader, or to an unswerving authoritarianism (e.g., cults; gangs).

We build $A$ with equation (1), then convert it to an orthogonal matrix. Let $A$ be a symmetric matrix with potential eigenvalues $\lambda_{1}, \ldots \lambda_{n}$. If $Q$ is an orthogonal matrix with real values and if $Q^{-1}=Q^{t}$, so that the inverse of $Q$ equals its transpose, then the row vectors (or column vectors) are orthogonal, and $Q^{t} A Q$ diagonalizes matrix $A$ into its eigenvalues.

Let $A$ be an operator on a social object, $\psi$, within its internal zone of influence; $\psi$ could be an agent or a team, etc.; and let $\psi$ be a column vector that represents the state of the social object as operator $A$ transforms state vector $\psi$ into a matrix. When $\psi$ is represented on two sides of an equation as

$$
A|\psi>=x| \psi>
$$

then $\lambda$ is a scalar that is the eigenvalue or characteristic of $A$, and $\psi$ becomes an eigenvector or eigenfunction. The usual way to solve for the eigenvalue, $\lambda$, is with an iterative process: $A \psi-\lambda I \psi=(A-\lambda I) \psi=0$, where $I$ is the identity matrix (i.e., $\left[\begin{array}{ll}1 & 0 \\ 0 & 1\end{array}\right]$ ).

The outer product of two state vectors is an operator; and the outer product of two eigenvectors is a special operator or projector, $P$. $P$ projects an unknown or arbitrary state vector, $\psi$, into an eigenfunction and eigenvalue. Eigenfunctions form a basis that is orthonormal; i.e., given eigenfunctions $\psi$ and $\phi$ and $\langle\psi \mid \phi\rangle$ as the inner product of the two eigenfunctions, then $\langle\psi \mid \phi\rangle=\psi_{1} \phi_{1}+\psi_{2} \phi_{2}+\ldots=\delta_{i j}$, where $\delta_{i j}$ as the Kronecker delta equals to 1 when $i=j$, otherwise 0 . This result means that state vectors are normalized, the inner product summing to 1 when the eigenvectors are the same (i.e., $\cos 0 \mathrm{deg}=1$ ); it also means that the probabilities of measuring interdependent (or bistable) factors always sums to 1 (i.e., when the probability of one bistable factor goes to zero, that of the other goes to 1 ).

Bistability. In contrast to a stable image, an example of bistability is easily apprehended by comparing a stable image with a bistable illusion (fig. 1).

If $\psi$ was a simple column vector representing the state of its independent elements, putting aside the mathematical manipulations to find the eigenvalues, there would be little ambiguity in constructing conceptual models or in understanding them based on what amounts to a convergent, rational process. Conceptual difficulties arise and intuition fails when interdependence (groupiness) is introduced. Be- ginning with simple bistability, $\psi$ becomes a superposition of two orthogonal but factorable states, such as an observation and an action; a republican and a democrat; or a single tribal ingroup and outgroup (e.g., [22]). Putting time evolution aside for this paper, we gain insight into a static situation by letting $\mid 0>$ be the name of a column vector that represents one of the orthogonal factors of a basis, and I1 > the other (e.g., we arbitrarily set observation to $10>=\left[\begin{array}{l}1 \\ 0\end{array}\right]$, and action to $I 1>=\left[\begin{array}{l}0 \\ 1\end{array}\right]$ ); similarly, we could let a single person oscillate between being a conservative, represented by $\mid 0>$, and a liberal, represented by $\mid 1>$ (and vice versa); or ingroup $A$ versus outgroup $B$ ([17]). Orthogonal vectors $10>$ and $\mid 1>$ form a basis.
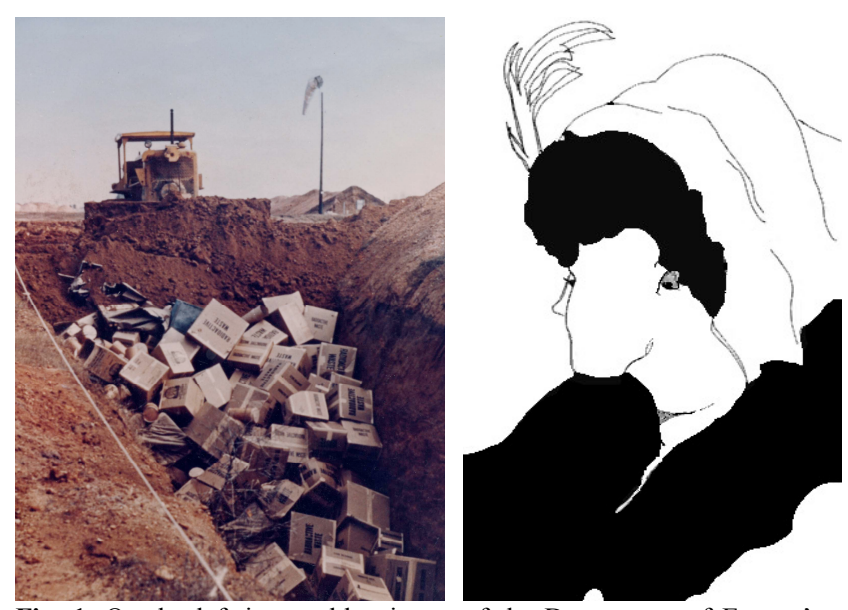

Fig. 1. On the left is a stable picture of the Department of Energy's (DOE) waste management operations using cardboard boxes, its primary container to dispose of solid radioactive wastes until the mid-1980s, a practice immediately suspended after it was publicized ([20]). On the right is a bistable illusion of either an old woman looking downward and to the left, or of a young woman looking over her right shoulder. Cacioppo and colleagues [21] established that bistable images cannot be held in awareness simultaneously. Generalizing, the presence of two independent images or concepts requires a focus on one that precludes a simultaneous focus on the other. ${ }^{1}$

Incompleteness and uncertainty. New information may serve to change an individual's strongest beliefs; however, confirmation bias makes it unlikely that contradictory new information will be judged objectively by a committed believer [23]. The avoidance of cognitive dissonance keeps the most important attitudes and beliefs of humans stable [24], indicating that effort is necessary to change strongly held beliefs [25], foreshadowing the conflict that spontaneously arises between political parties, placing a premium on a buffer between them, such as a neutral middle class; when the neutral middle dissipates, open conflict between the extremes in a society becomes likely [26]. Together, these

\footnotetext{
${ }^{1}$ From the research of U. Neisser: For a brain with finite computing power, zooming in to focus on one thing always means picking up less information about everything else". Scientific American (2013, 6/11), "Your Hidden Censor: What your mind will not let you see".
} 
two biases make it unlikely that a leader of say a conservative or liberal group would entertain an opposing (orthogonal) viewpoint, especially when entertaining such a view threatened the power, status or access to resources under control. Not so for those located farthest from a leadership position or removed from the direct control of a funding source, so called "neutral" members or swing voters, those whose beliefs are quixotic, unstable or malleable. To simplify what constitutes a complexity of its own, assume there are ideologues on either side of an issue, and that all others are swing voters ensconced in the neutral camp. For a single individual in a political swing camp, we postulate that both views are held simultaneously in a state of superposition; likewise for those individuals mindful while in a state of complicated action (viz., possessing a competent grasp of reality, not daydreaming, or not in a state of denial about their action, as might be true for an alcoholic in denial). For a single social agent in a superposition of orthogonal factors (opposed beliefs; or beliefs and actions), we propose:

$$
|\psi>=a| 0>+b \mid 1>
$$

with the basis for a single agent written as $\{|0>| 1>$,$\} , where$ $\left|a^{*} a^{\prime}\right|=a^{*} a=a^{2}$ (here $a^{\prime}$ is the complex conjugate that we use to represent the oscillations caused by an illusion) gives the probability of a social object being found in state $\mid 0>$, and with $b^{2}$ giving the probability of being in state $\mid 1>$. But this state vector is still factorable, suggesting that the oscillating (conflicting) perspectives for a neutral individual may be simply aggregated to reconstruct the oscillation (but see below).

Equation (3) is easily factored; however, breaking apart a bistable state of superposition leads to a loss of information, producing incompleteness about the interdependent state.

Evidence of incompleteness for individuals: Hypothesis: The effect of measuring $a$ in equation 3 produces incomplete information about the measurement of $b$.

The evidence from studies of individuals: First, Tversky [27] found that an individual's justification for an action is unrelated to the action performed. Second, despite the reports by women of taking HIV prevention pills $95 \%$ of the time, the measure of effective drug levels in their blood near the time of infection was less than $26 \%{ }^{2}$ Third, in a 30 -year meta-analysis, the association was found to be negligible both between self-esteem and academic success, and between self-esteem and success at work [28].

Interdependence. To model a group in a state of interdependence, we introduce the tensor product of independent elements, for example, $|0>\otimes| 0>$, represented as $\mid 00>$; and $|1>\otimes| 1>$, represented as $\mid 11>$. The basis for a 2 agent system becomes $\{|00>, 01>| 10>,, \mid 11>\}$. Factorability means independent objects, that any separable vector space $V$ as by tensor decomposition into basis elements is not interdependent. i.e., given the state vector $\mid \psi>$ of a system, where $V=V_{1} \otimes V_{2} \otimes \ldots \otimes V_{n}$, the state $|\psi\rangle$ is separable iff $|\psi\rangle=v_{1} \otimes v_{2} \otimes \ldots \otimes v_{n}$.

\footnotetext{
${ }^{2}$ News, 2012, Science, 335: 1291.
}

Otherwise, $\mid \psi>$ is in an interdependent state. For example, when

$$
\mid \psi>=\frac{1}{\sqrt{2}}(|00>+| 11>)
$$

$|\psi\rangle$ is in a state of interdependence. To prove, let $\left(a_{1}\left|0>+b_{1}\right| 1>\right)\left(a_{2}\left|0>+b_{2}\right| 1>\right)=\frac{1}{\sqrt{2}}(|00>+| 11>)$. On the left-hand side, there exists no combination of $a$ 's and $b$ 's such that $a_{1} b_{2}$ and $b_{1} a_{2}$ are both zero. Equation (3) reflects two agents in a state of superposition. Breaking it into separable elements not only loses information, but also produces an incomplete state that cannot be recombined to reproduce the state of superposition.

Evidence of incompleteness for groups: Hypothesis: Individuals are poor at multitasking [10]. In contrast, the function of a group is to multitask. Multitasking with agent-1 and agent- 2 forces them to focus on their individual tasks to manage the work-flows and communications between them to constitute the elements of a multitask, reducing the information available to them about their own performances.

The evidence from studies of organizations: First, Bloom and colleagues [29] found that the estimation by managers of their firm's performance was unrelated to the firm's actual performance. Second, Lawless and colleagues [20] found no association between book knowledge of air combat skills and performance in combat, but a significant association between training and performance. Third, uncertainty in the observations of better-run organizations was found to become noise (e.g., compared to say a hostile merger, the Mara Salvatrucha or MS-13 gang expends free energy to become relatively dark on purpose; in contrast to MS-13, Apple is dark by successful teamwork; in [12]). Fourth, game preferences made before games played by humans in dyads or groups were found to be unrelated to the game choices they made during games [30]. Fifth, despite that most mergers fail $[44],{ }^{3}$ they are often pursued to offset a vulnerability, ${ }^{4}$ to gain a new technology, to remove a competitor, but also to transform a business model.

Finally, combining experiences for individuals and groups, among the numerous common examples of incompleteness that could be offered: Couples therapy is very difficult for therapists. ${ }^{5}$ Almost all team sports, and many great golfers, use coaches; many successful firms use con-

\footnotetext{
${ }^{3}$ For many years, H.P. has been acquiring firms through mergers. Recently, however, H.P. is headed towards collapse, with its parts worth more than the whole company, a split up or spinoff makes sense: H.P.'s market capitalization is about $\$ 27$ billion. That's scarcely more than half the price its parts would fetch as standalone entities." (From the New York Times (2012, 12/6), "Why breaking up H.P. makes sense".) ${ }^{4}$ CNBC (2013, 2/20), "Office Depot, Office Max plan \$1.2 Billion merger deal. ... The two companies are competitors to Staples, the world's largest supplier of office goods who dominates the space. The deal appears to be an attempt by both ... to become more competitive ... even as they confront declining market share and consumers that move to buy online."

${ }^{5}$ e.g., New York Times (2012, 3/2), Does couple therapy work?
} 
sultants; and military team sorties end only after the lead pilot debriefs the team. And even though there are no satisfactory theories of team performance [5], it has been found that team performance in aviation is improved with practice [4]. Similarly, the Institute of Medicine concluded that adopting the example of aviation's team training would reduce errors in medicine [31].

In sum, as Galton discovered when a crowd of independent individuals was able to accurately estimate the weight of an ox, groups that process all of the available information are more likely than any one individual to be correct. ${ }^{6}$

Competing groups and limit cycles. We postulate that at the level of individuals and groups, there is a constant competition to focus on the orthogonal functions for observation and action, orthogonal views like conservatism and liberalism, or orthogonal membership in tribe $A$ or tribe $B$. The competition between these orthogonal functions results in limit cycles ${ }^{7}$ (e.g., for data on the competition between A ple and Samsung, see Fig.2).

\section{L-V Notional Chart}

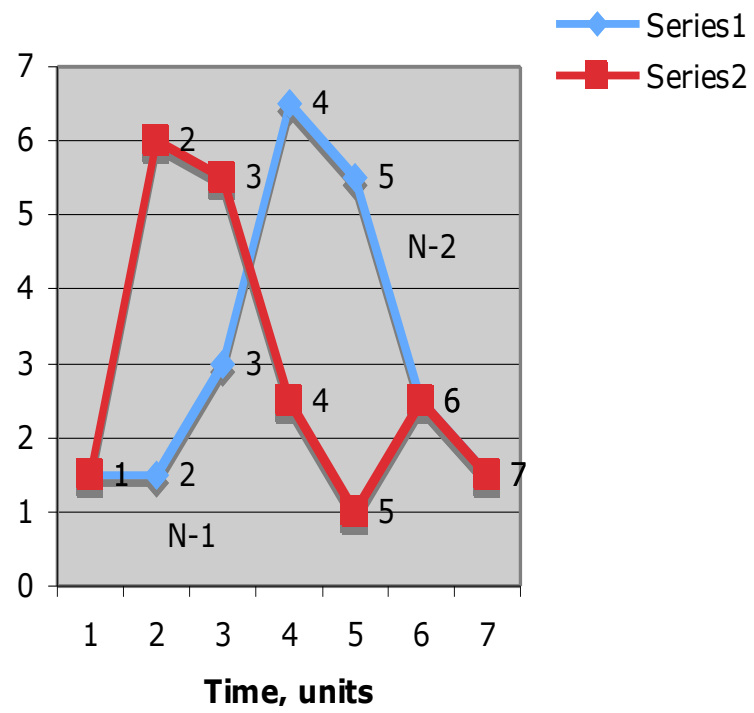

\footnotetext{
${ }^{6}$ This idea is still current; e.g., Surowiecki's (2004) The Wisdom of Crowds; conversely, it is an effect that is lost under the combination of isolation and authoritarianism to produce stagnation, possibly reversing social evolution; see M. Ridley's review of the present dire straits of North Korea in "From Phoenicia to Hayek to the 'Cloud', in Wall Street Journal $(2011,9 / 11)$. Similar stagnation is seen in varying degrees in Cuba, Russia, and others, like Venezuela; e.g., Bloomberg (2013, 1/9), "Food Shortages in Venezuela Bigger Worry Than Constitution".

${ }^{7}$ A limit cycle is the trajectory of a dynamical system's oscillations of periodic behavior(s) caused by the interdependence between at least 2 factors; they can be stable; and they can be beneficial; e.g., New York Times (2012, 9/28), "Why the beaver should thank the wolf". Limit cycles reflect a "gap" in social reality, explained in the text.
}

\section{President Obama's Approval (RCP)}

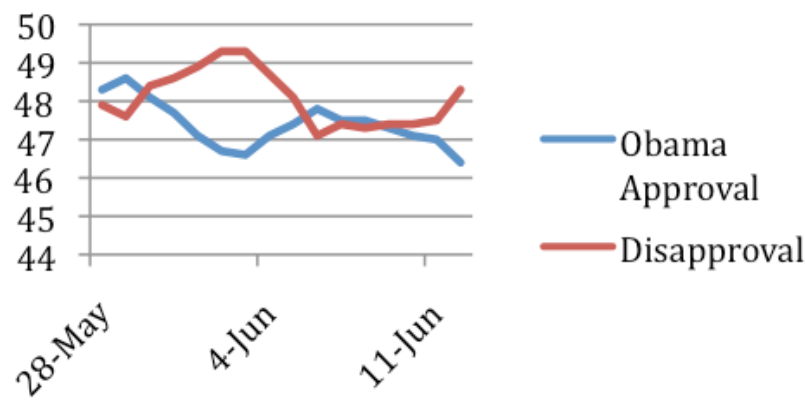

Fig. 2A (Top). Bistable data (from a simple coupled equation) generate a limit cycle, $N_{1}$ versus $N_{2}$; in [16]) as displayed over time, $t$. Notional parameters produce "frictionless" oscillations. We interpret $N_{l}$ and $N_{2}$ to reflect competition at time 1 (and $t=3.5,6$ and 7) (from [12], p. 296).

Fig. 2B (Below). From RCP, ${ }^{8}$ President Obama's daily poll approval numbers suggests a limit cycle.

Limit cycles depend on the free flow of neutrals to one (ideological, commercial, scientific, etc.) belief position or another. Limit cycles can be suppressed under authoritarian rule. In a dictatorship, control is asserted by censoring information [16]; i.e., by forcibly setting $a$ or $b$ to zero. But while control is gained, the opportunity for mistakes increases dramatically (e.g., DOE's mismanagement of nuclear wastes prior to $1985 ;{ }^{9}$ China's air ${ }^{10}$ and water ${ }^{11}$ contamination today; USS Vincennes shoot-down of an Iranian airbus in 1988, killing all aboard; ${ }^{12}$ and USS Greeneville's collision with a Japanese fishing boat, ${ }^{13}$ killing nine aboard).

Compared to a collection of independent individuals, we had initially assumed that the entropy $(S)$ is set to zero for a perfect team, the driving motivation to form a tribe. We now justify this assumption in the limit as follows (and contradict it later). Transaction costs are lower for individuals inside of a firm performing the same functions as for those same individuals multitasking in a firm [32]. This cost differential motivates the six-sigma processes designed to reduce waste in a firm, thereby precluding the tradeoffs a firm must make to find the new sources of free energy needed to adapt or to innovate [3], unexpectedly generating more entropy in a changing environment [2], and setting a firm up for failure. ${ }^{14}$

Equation (2) does not allow us to capture tradeoffs. For two operators, we begin by writing:

\footnotetext{
${ }^{8}$ Data from RCP: http://www.realclearpolitics.com/epolls/other/president_obama_job_ approval-1044.html

${ }^{9}$ New York Times $(1985,3 / 17)$, "Living with nuclear waste".

${ }^{10}$ New York Times $(2013,1 / 14)$, "China lets media report on air pollution crisis."

${ }^{11}$ Al Jazeera (2013, 3/4), "China comes clean on water pollution".

${ }^{12}$ Washington Post (1988, 7/4), "Navy missile downs Iranian Jetliner."

${ }^{13}$ Chicago Tribune (2001, 11/26), "3 investigating Admirals tour USS Greeneville's control room."

${ }^{14}$ e.g., the collapse of sales of the Blackberry by RIM; from Wall Street Journal (2012, 3/30), "RIM weighs bleak options".
} 


$$
[A, B]=A B-B A \text {. }
$$

When two operators representing two different tribes have the same eigenvalue, then the operators commute: $[A, B]=A B-B A=0$. With agreement between two erstwhile competitors, ${ }^{15}$ the combined social system is stable, no oscillations occur, nor do limit cycles exist. But when disagreement arises between two competitors, their two operators do not commute, and $[A, B]=i C$, where $C$ is a measure of the gap or distance in reality between $A$ and $B$. However, as multitasking improves, the tradeoffs between each group's focus on the tasks at hand interfere with their meta-perspectives on how best to change or tune tasks to improve performance [29], motivating the tradeoffs that reflects the conservation of information (from [33]):

$$
\sigma_{A} \sigma_{B} \geq 1 / 2
$$

where $\sigma_{A}$ is the standard deviation of variable $A$ over time, $\sigma_{B}$ is the standard deviation of its Fourier transform, introducing frequency, and the two together form a Fourier pair that reflect tradeoffs. Interdependent uncertainty generates tradeoffs. For example, as uncertainty in a team's or firm's skills decrease, uncertainty in its observations increase.

"Gaps" in the construction of reality: Equations (2) and (4) reflect the existence of a "gap" in social reality, $C$, that permits social dynamics to operate. Social dynamics derive from the challenges to claims, social illusions [34] and irrationality [35], feeding limit cycles (May, 1973). The evidence indicates that the conscious awareness of signals takes about 500 msecs, but under decision-making, it can extend to several seconds (of at least 7 secs) before a human's consciousness becomes aware of its "desire" to switch to a new choice (Bode et al., 2011) that can then be articulated by the human brain's running narrator [37], ${ }^{16}$ often construed as "free will". 17

Gaps are needed to create a state of superposition over a claim, to process the challenges that establish the oscillations between claims, giving observers time and space to process sequentially the information derived from opposing perspectives. But the human motivation is to believe that knowledge processing is too cumbersome, leading to the various illusions such as that a merger reduces overcrowding in a collapsing market, that six-sigma processes safely improve profits, or that market returns improve by chasing market leaders. The motivation in these and other illusions is to

\footnotetext{
${ }^{15}$ For example, see agreement between the New York Times and Wall Street Journal over the pigs found in the river headed to Shanghai; cf. New York Times (2013, 3/12), "China: Dead pigs in river near 6,000"; and Wall Street Journal, Review \& Outlook, Editorial (2013, 3/15), "What's in China's water? Of floating pigs and political change."

${ }^{16}$ Working in the field with teams of robots capable of collectively updating their status, changing roles among their members, and making re-tasking decisions, all in less that one second, the anecdotal evidence indicates that their human operators should not be sent alerts in less than 45 seconds and preferably 90 seconds.

${ }^{17}$ Raghunathan, R. (2012, 5/8), "Free will is an illusion, so what?", Psychology Today.
}

ignore, reduce or replace the "gaps" in reality with a rational approach instead of an emphasis (focus) on problem solutions. For example, McKelvey and colleagues ([1] p. 156) have concluded that "Microsoft's ecosystem is a good example of SOC [self-organized criticality] working well across an entire industry ... [that] is PL [power law] distributed. Given that well-working SOC dynamics are required for industry change, growth and survival the PLs become good indices of whether or not an industry is well-endowed with SOC dynamics." But McKelvey's notions of SOC and PLs are descriptive and a-causal, which are unable to capture the recent market failures of Microsoft. ${ }^{18}$

\section{Discussion}

Despite the accumulating evidence against the traditional model, it remains rational (e.g., Bayesian). Silver [38] concludes that the brain forms and continually updates a set of Bayesian "priors" learned over a lifetime used to interpret new data that corresponds to its environment. But Silver's technique of aggregating polling data copies Galton's insight. The more important question is why Democrats and Republicans look at the same data but interpret it differently at the same time, thereby generating bistable illusions, conflict and oscillations. Numerous examples exist; e.g., R.A. Fisher, the esteemed statistician and evolutionary biologist [39], argued against the evidence that smoking cigarettes would cause cancer; but Fisher was a smoker [40], likely the cause of his not accepting the available evidence.

\section{Conclusion:}

We have argued that interdependence combines with cognitive dissonance to make those of us who adopt strong beliefs act to suppress both our internal cognitive narrator, known as confirmation bias [23], but also the alternative views of our ingroup, forming the ingroup-outgroup bias [17]; when these beliefs are unchallenged, they give the illusion of stable reality; but when challenged, they drive the oscillations of social behavior between competing teams, tribes, or firms across a system [20]. Thus, the presence of alternative views in the decision process is not only the end of certainty that motivates tradeoffs (equations 3 and 5, respectively), but it is also the source of information that competition generates for observers to process that preclude, reduce or mitigate tragedies (e.g., no modern democracy has ever suffered from famine; in [41]; and no modern democracy has ever started a war against another democracy; in [42]). To defend an individual, Chagon [22] concluded that people find safety in numbers of their own. However, although not very popular to any single tribe of Republicans or Democrats, competing religions or different races, nonetheless, it is the competition

\footnotetext{
${ }^{18}$ Despite its leadership with computer desktop software, Microsoft has failed to successfully develop a mobile device or tablet computer; from Bloomberg $(2013,315)$, "Microsoft's surface tablet is said to fall short of predictions"; and
} 
for the strongest idea that has become the modern foundation of free speech [43]. ${ }^{19}$

Without competition, information incompleteness impedes social evolution. But with conflict and its management, indirect control of hybrid teams may be feasible. Social uncertainty spontaneously generates interdependence, just as interdependence generates social uncertainty. Both require a "gap" in reality that promotes competition as neutrals sort through the interpretations when they are free to make the best choice, switching back when a choice does not pan out, forming limit cycles that indirectly provide social control. Social information must remain incomplete, forcibly true under dictatorships to maintain direct control [16], but inescapably true in democracies with working checks and balances. However, unlike dictatorships, the search for completeness in democracies leads to social evolution.

Future research. Finally, we began by setting the baseline entropy for well-functioning teams to zero [12]. Next, we need to revise it to underscore the cognitive difficulty implied by equation (3) for two or more agents multitasking together in a state of superposition. Equation (3) suggests on the one hand how a team or an organization can perform at a high level, but also why on the other hand they are incomplete witnesses of their performance. To close out our project, how can agents generate the data in Figure 2 for equation (3) or (4)? We suspect that a conflict center creates interference among superposed neutrals; that winning a debate or selling more Apple computers on one day somewhat suppresses a conflict center's complementary element, producing stable results; that a tie causes no movement in the results; and that a more competitive counterattack from a previously failing candidate or firm creates the return arm in the results that builds the limit cycle.

Acknowledgements: This material was based upon work supported by, or in part by, the U.S. Army Research Laboratory and the U. S. Army Research Office under contract/grant number W911NF-10-1-0252.

\footnotetext{
${ }^{19}$ In his dissenting opinion, Justice Holmes wrote: "when men have realized that time has upset many fighting faiths, they may come to believe even more than they believe the very foundations of their own conduct that the ultimate good desired is better reached by free trade in ideas -- that the best test of truth is the power of the thought to get itself accepted in the competition of the market, and that truth is the only ground upon which their wishes safely can he carried out."
} 


\section{References}

[1]. McKelvey, B. Salmador, M.P., Morcillo, P. \& Rodriguez-Anton, M. (2013), Towards an econophysics view of intellectual capital dynamics: from self-organized criticality to the stochastic frontier, Knowledge Management Research \& Practice, 11: 142-161.

[2]. Conant, R. C., \& Ashby, W.R. "Every good regulator of a system must be a model of that system." International Journal of Systems Science 1(2): 899-897 (1970).

[3]. Christensen, C. M. The innovator's dilemma (6-sigma) (2011).

[4]. Salas, E., Diaz-Granados, D., Klein, C., Burke, C.S., Stagl, K.C., Goodwin, G.F. \& Halpin, S.M., "Does Team Training Improve Team Performance?", Human Factors, 50(6): 903-933 (2008).

[5]. Bell, B. S., Kozlowski, S.W.J. \& Blawath, S. Team Learning: A Theoretical Integration and Review. The Oxford Handbook of Organizational Psychology. Steve W. J. Kozlowski (Ed.). New York, Oxford Library of Psychology. Volume 1 (2012).

[6]. Smith, W. K., \& Tushman, M.L. "Managing strategic contradictions: A top management model for managing innovation streams." Organizational Science 16(5): 522-536 (2005).

[7]. Lawless, W.F., Llinas, J., Mittu, R., Sofge, D., Sibley, C., Coyne, J. \& Russell, S. Robust Intelligence (RI) under uncertainty: Mathematical and conceptual foundations of autonomous hybrid (human-machine-robot) teams, organizations and systems, Structure \& Dynamics, 6(1) (2013).

[8]. Jamshidi, M. Control of system of systems. Intelligent Control Systems with an introduction to System of Systems. T. Nanayakkara, Sahin, F., \& Jamshidi, M. London, UK, Taylor \& Francis Publishers. 2(8) (2009).

[9]. Schweitzer, F., Fagiolo, G., Sornette, D., Vega-Redondo, F., Vespignani, A., \& White, D.R. "Economic networks: The new challenges." Science 325: 422-425 (2009).

[10]. Wickens, C. D. Engineering psychology and human performance $\left(2^{\text {nd }}\right.$ ed.). Merrill Publishing (1992).

[11]. Ambrose, S. H. "Paleolithic technology and human evolution." Science 291: 1748-1753 (2001).

[12]. Lawless, W. F., Angjellari-Dajci, Fjorentina, Sofge, Donald A., Grayson, James, Sousa, José Luis \& Rychly, Laura, A New Approach to Organizations: Stability and Transformation in Dark Social Networks, Journal of Enterprise Transformation, 1:4, 290322 (2011).

[13]. Conant, R. C. "Laws of information which govern systems." IEEE Transaction on Systems, Man, and Cybernetics 6: 240-255 (1976).

[14]. Bowles, S. "Warriors, levelers, and the role of conflict in human evolution." Science(336): 876-879 (2012).

[15]. Darwin, C. The descent of man New York, Appleton (1973).

[16]. May, R. M.. Stability and complexity in model ecosystems. Princeton, NJ, Princeton University Press (1973/2001).

[17]. Tajfel, H. "Experiments in intergroup discrimination." Scientific American 223(2): 96-102 (1970).

[18]. Chagnon, N. A. "Life histories, blood revenge, and warfare in tribal populations." Science 239: 985-992 (1988).

[19]. Kenny, D. A., Kashy, D.A., \& Bolger, N. Data analyses in social psychology. Handbook of Social Psychology. D. T. Gilbert, Fiske, S.T. \& Lindzey, G., McGraw-Hill. 4th Ed., 1: pp. 233-65 (1998).

[20]. Lawless, W. F., Rifkin, S., Sofge, D.A., Hobbs, S.H., AngjellariDajci., F., Chaudron, L. \& Wood, J. "Conservation of Information: Reverse engineering dark social systems." Structure and Dynamics 4(2) (2010).

[21]. Cacioppo, J. T., Berntson, G.G., \& Crites, S.L., Jr., Ed. Social neuroscience: Principles, psychophysiology, arousal and response. Social Psychology Handbook of Basic Principles. Guilford (1996).

[22]. Chagnon, N. A. The Yanomamo. New York, Wordsworth (2012).

[23]. Darley, J. M. \& Gross, P.H. A Hypothesis-Confirming Bias in Labelling Effects. Stereotypes and prejudice: essential readings. C. Stangor, Psychology Press, p. 212 (2000).

[24]. Festinger, L., A theory of cognitive dissonance, Stanford U. (1985).

[25]. Cooper, J., Cognitive dissonance: 50 years of a classic theory. London: Sage publications (2007).

[26]. Kirk, R. (2003). More terrible than death. Massacres, drugs, and America's war in Columbia, Public Affairs.

[27]. Shafir, E., \& LeBoeuf, R.A. "Rationality." Annual Review of
Psychology 53: 491-517 (2002).

[28]. Baumeister, R. F., Campbell, J.D., Krueger, J.I., \& Vohs, K.D. "Exploding the self-esteem myth." Scientific American (2005, January).

[29]. Bloom, N., Dorgan, S., Dowdy, J., \& Van Reenen, J. "Management practice and productivity." Quarterly Journal of Economics 122(4): 1351-1408 (2007).

[30]. Kelley, H. H. "Lewin, situations, and interdependence." Journal of Social Issues 47: 211-233 (1992).

[31]. Kohn L, Corrigan J, Donaldson M., To err is human: building a safer health system. Committee on Quality of Health Care in America. Washington, DC: Institutes of Medicine. National Academy Press (2000).

[32]. Coase, R. The nature of the firm, Economica 4: 386 (1937).

[33]. Cohen, L. Time-frequency analysis: theory and applications, Prentice Hall (1995).

[34]. Adelson, E. H. Lightness perceptions and lightness illusions. The new cognitive sciences, 2nd Ed. M. Gazzaniga. MIT Press (2000).

[35]. Kahneman, D. Thinking, fast and slow. New York, MacMillan (Farrar, Straus \& Giroux) (2011).

[36]. Bode, S., He, A.H., Soon, C.S., Trampel, R., Turner, R. \& Haynes, J.R., Tracking the unconscious generation of free decisions using ultra-high field fMRI, PLoS One, 6(6): e21612.

[37]. Gazzaniga, M.S., Who's in charge? Free will and the science of the brain. New York; Ecco (2011).

[38]. Silver, N., The signal and the noise. Why so many predictions fail-But some don't/The art and science of prediction. New York: Penguin (2012).

[39]. Box, J.A. (1978, R.A. Fisher: The life of a scientist, New York: Wiley (2011).

[40]. Stolley, P.D., American Journal of Epidemiology, 133: 416 (1991).

[41]. Sen, A. Development as freedom, Knopf (2000).

[42]. Wendt, A. Social theory of international politics, Cambd. U. (1999).

[43]. Holmes, O. W. Dissent: Abrams v. United States (1919).

[44]. Andrade, G., \& Stafford, E. Investigating the economic role of mergers (Working Paper 00-006). Cambridge, MA; Retrieved from http://ssrn.com/abstract=47264, Harvard (1999). 